\title{
TESTS OF THE BINARY HYPOTHESIS OF NOVAE THROUGH NOVA NEBULAE OBSERVATIONS
}

\author{
J. B. HUTCHINGS \\ Dominion Astrophysical Observatory, Victoria, B.C., Canada
}

\begin{abstract}
I should like to follow up Dr Schatzman's talk with a few words on models of Nova nebulae. Geometrical models for novae Aql 1921 and Her 1934 have been derived by Mustel and Boyarchuk (1970), for novae Del 1967, Vul 1968 and Ser 1970 by Hutchings (1972), for Aql 1921 by Weaver (1974) and for Del 1967 by Malakpur (1973). Very different techniques and lines of reasoning have been used by all these people, but the models derived all have very strong similarities which should lead us to take them seriously in considering the nature of the nova mechanism.

It appears that nova remnants are characterized by two polar cones or blobs of similar but opposite velocity and similar density, moving along the axis of rotation of the system. In addition, one or more rings of matter may exist symmetrically about the equatorial plane, with a recession velocity and density which, in general, are different from the polar matter. The polar blobs appear to be associated with the matter giving rise to the 'principal' spectrum in the bright phases of the nova.

Three possible main causes have been suggested for the formation of such remnants, all connected with the initial outburst:(1) Weaver has proposed that an initially spherically symmetrical expansion is impeded and deflected by matter circulating in an accretion ring around the degenerate star. (2) Schatzman has suggested that the presence of the ring may favour an initial outburst preferentially in the polar regions. (3) Hutchings has suggested that physical interference of an initially spherical expansion may occur by a secondary component whose size is a large fraction of the separation of the two stars.

I feel that these points need to be considered by theoreticians in proposing detailed mechanisms of the nova outburst.
\end{abstract}

\section{References}

Hutchings, J. B.: 1972, Monthly Notices Roy. Astron. Soc. 158, 177.

Malakpur, I.: 1973, Astron. Astrophys. 24, 125.

Mustel, E. R. and Boyarchuk, A. A.: 1970, Astrophys. Space Sci. 6, 183.

Weaver, H.: 1974, in G. Contopoulos (ed.), Highlights of Astronomy, 3, XVth General Assembly of the IAU, D. Reidel Publishing Company, Dordrecht, Holland, p. 423. 